ORNL/TM-12988

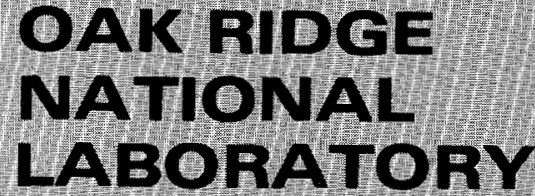

\section{MARTRW MARUETRA}

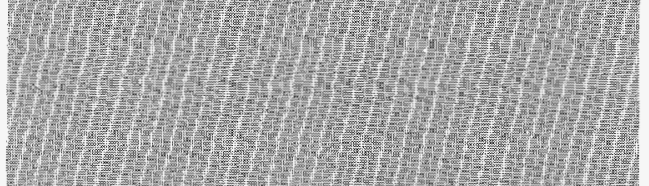

W.

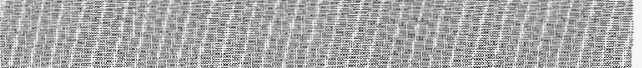

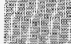

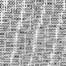

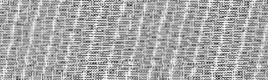

thet

Whe

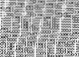

(a)

1.:

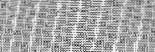

4.

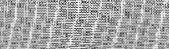

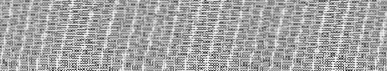

In:

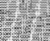

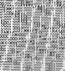

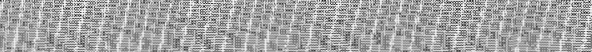

14.

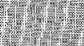

4he

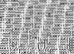

(1).

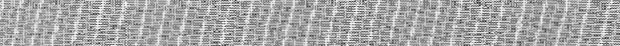

(2)

(1)

4 1 (1)

14.

MAVAGED BY

MARTIW MARIETTA ENERGY SYSTEMS, NC.

FOR THE UNITEO STATES

DEPABTMENT OF ENERGY
A NOTE ON

SEVEN ANALOGOUS PROPERTIES BETWEEN STIRLING NUMBERS OF THE FIRST KIND AND BINOMIAL COEFFICIENTS

Tommy Wright

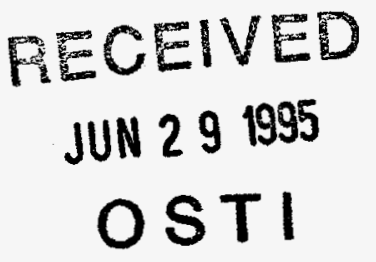

DISTHBBUTION OF THIS DOCUMENT IS UNLIMITED 
This report has been reproduced directly from the best available copy.

Available to DOE and DOE contractors from the Office of Scientific and Technical Information, P.O. Box 62, Oak Ridge, TN 37831; prices available from (615) 576-8401, FTS 626-8401.

Available to the public from the National Technical Information Service, U.S. Department of Commerce, 5285 Port Royal Rd., Springfield, VA 22161.

This report was prepared as an account of work sponsored by an agency of the United States Government. Neither the United States Government nor any agency thereof, nor any of their employees, makes any warranty, express or implied, or assumes any legal liability or responsibility for the accuracy, completeness, or usefulness of any information, apparatus, product, or process disclosed, or represents that its use would not infringe privately owned rights. Reference herein to any specific commercial product, process, or service by trade name, trademark, manufacturer, or otherwise, does not necessarily consti tute or imply its endorsement, recommendation, or favoring by the United States Government or any agency thereof. The views and opinions of authors expressed herein do not necessarily state or reflect those of the United States Government or any agency thereof. 


\section{DISCLAIMER}

Portions of this document may be illegible in electronic image products. Images are produced from the best available original document. 
Computer Science and Mathematics Division

Mathematical Sciences Section

\title{
A NOTE ON \\ SEVEN ANALOGOUS PROPERTIES BETWEEN \\ STIRLING NUMBERS OF THE FIRST KIND \\ AND BINOMIAL COEFFICIENTS
}

\author{
Tommy Wright \\ Mathematical Sciences Section \\ Oak Ridge National Laboratory \\ P. O. Box 2008 Bldg 6012 \\ Oak Ridge, Tennessee 37831-6367
}

Date Published: June 1995

Research was supported by the
ASA/NSF/Census Research Fellow Program
of the U. S. Bureau of the Census.

Prepared by the

Oak Ridge National Laboratory

Oak Ridge, Tennessee 37831

managed by

Martin Marietta Energy Systems, Inc.

for the

U. S. DEPARTMENT OF ENERGY

under Contract No. DE-AC05-84OR21400 

TABLE OF CONTENTS

ABSTRACT $v$

1. INTRODUCTION 1

2. SOME ANALOGOUS PROPERTIES OF THE COEFFICIENTS $\left[\begin{array}{l}n \\ r\end{array}\right]$ and $\left(\begin{array}{l}n \\ r\end{array}\right) \quad 1$ ACKNOWLEDGMENT

REFERENCES 


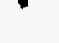




\author{
A NOTE ON \\ SEVEN ANALOGOUS PROPERTIES BETWEEN \\ STIRLING NUMBERS OF THE FIRST KIND \\ AND BINOMIAL COEFFICIENTS \\ Tommy Wright
}

\begin{abstract}
This notes gives seven analogous properties between Stirling numbers of the first kind and binomial coefficients.
\end{abstract}




\section{INTRODUCTION}

If $n$ and $r$ are both nonnegative integers where $r \leq n$, the binomial coefficient $\left(\begin{array}{l}n \\ k\end{array}\right)$ is given by

$$
\left(\begin{array}{l}
n \\
r
\end{array}\right) \equiv \frac{n !}{r !(n-r) !} .
$$

The symbol $\left(\begin{array}{l}n \\ r\end{array}\right)$ is called a binomial coefficient because it is the coefficient of the $(r+1)^{\text {th }}$ term in the expansion of $(1+x)^{n}$ by the binomial theorem. Furthermore, these coefficients are the entries in Pascal's triangle. For a recent historical treatment of Pascal's arithmetical triangle's roots, which stretch backward before Christ, see Edwards(1987). The binomial coefficient plays a fundamental role in several areas including combinatorics, applied probability, and probability sampling (Knuth, 1981; Graham, Knuth, and Patashnik, 1989; Ross, 1989; Wilf, 1989; and Wright, 1989, 1991).

If $n$ and $r$ are both nonnegative integers where $r \leq n$, the Stirling Number of the First Kind $\left[\begin{array}{l}n \\ r\end{array}\right]$ is defined as

$$
\left[\begin{array}{l}
n \\
r
\end{array}\right] \equiv \text { the sum of all possible products of } n-r \text { integers taken from the first } n \text { positive integers. }
$$

For $r=n$, we define $\left[\begin{array}{l}n \\ n\end{array}\right] \equiv 1$. Note that $\left[\begin{array}{l}n \\ 0\end{array}\right] \equiv n !$. Thus if $n=4$ and $r=2,\left[\begin{array}{l}4 \\ 2\end{array}\right]=$ $1 \cdot 2+1 \cdot 3+1 \cdot 4+2 \cdot 3+2 \cdot 4+3 \cdot 4=35$. Also $\left[\begin{array}{l}4 \\ 0\end{array}\right]=4 !=24$ and $\left[\begin{array}{l}4 \\ 4\end{array}\right]=1$. In general, the number of terms in the sum $\left[\begin{array}{c}n \\ r\end{array}\right]$ is $\left(\begin{array}{l}n \\ r\end{array}\right)$.

In a result analogous to the binomial theorem, it can be shown that

$$
\prod_{i=1}^{k}(i+x)=\sum_{r=0}^{k}\left[\begin{array}{l}
k \\
r
\end{array}\right] x^{r} .
$$

The quantities $\left[\begin{array}{l}n \\ r\end{array}\right]$ have a triangular arrangement which is similar to Pascal's triangle for the binomial coefficients. (Graham, Knuth, and Patashnik (1989); and Wright(in press))

\section{SOME ANALOGOUS PROPERTIES OF THE COEFFICIENTS $\left[\begin{array}{l}n \\ r\end{array}\right]$ AND $\left(\begin{array}{l}n \\ r\end{array}\right)$}

In this section, we list several properties of the coefficients $\left[\begin{array}{l}n \\ r\end{array}\right]$. For each property, an analogous result is noted for Pascal's triangle. The proofs of these properties are straightforward.

Property 1.

$$
\left[\begin{array}{l}
n \\
r
\end{array}\right]=n\left[\begin{array}{c}
n-1 \\
r
\end{array}\right]+(n-1)\left[\begin{array}{l}
n-2 \\
r-1
\end{array}\right]+\cdots+(n-r)\left[\begin{array}{c}
n-(r+1) \\
0
\end{array}\right]
$$


Example 1.

$$
\left[\begin{array}{l}
5 \\
2
\end{array}\right]=5\left[\begin{array}{l}
4 \\
2
\end{array}\right]+4\left[\begin{array}{l}
3 \\
1
\end{array}\right]+3\left[\begin{array}{l}
2 \\
0
\end{array}\right]
$$

Analogous Property and Example:

$$
\begin{aligned}
& \left(\begin{array}{l}
n \\
r
\end{array}\right)=\left(\begin{array}{c}
n-1 \\
r
\end{array}\right)+\left(\begin{array}{l}
n-2 \\
r-1
\end{array}\right)+\left(\begin{array}{l}
n-3 \\
r-2
\end{array}\right)+\cdots+\left(\begin{array}{c}
n-(r+1) \\
0
\end{array}\right) . \\
& \left(\begin{array}{l}
5 \\
2
\end{array}\right)=\left(\begin{array}{l}
4 \\
2
\end{array}\right)+\left(\begin{array}{l}
3 \\
1
\end{array}\right)+\left(\begin{array}{l}
2 \\
0
\end{array}\right) .
\end{aligned}
$$

Property 2.

$$
\left[\begin{array}{l}
n \\
r
\end{array}\right]=\left[\begin{array}{l}
n-1 \\
r-1
\end{array}\right]+n\left[\begin{array}{l}
n-2 \\
r-1
\end{array}\right]+n(n-1)\left[\begin{array}{l}
n-3 \\
r-1
\end{array}\right]+\cdots+n(n-1) \cdots(r+1)\left[\begin{array}{l}
r-1 \\
r-1
\end{array}\right]
$$

Example 2.

$$
\left[\begin{array}{l}
5 \\
2
\end{array}\right]=\left[\begin{array}{l}
4 \\
1
\end{array}\right]+5\left[\begin{array}{l}
3 \\
1
\end{array}\right]+5 \cdot 4\left[\begin{array}{l}
2 \\
1
\end{array}\right]+5 \cdot 4 \cdot 3\left[\begin{array}{l}
1 \\
1
\end{array}\right]
$$

Analogous Property and Example:

$$
\begin{aligned}
& \left(\begin{array}{l}
n \\
r
\end{array}\right)=\left(\begin{array}{l}
n-1 \\
r-1
\end{array}\right)+\left(\begin{array}{l}
n-2 \\
r-1
\end{array}\right)+\left(\begin{array}{l}
n-3 \\
r-1
\end{array}\right)+\cdots+\left(\begin{array}{l}
r-1 \\
r-1
\end{array}\right) \\
& \left(\begin{array}{l}
5 \\
2
\end{array}\right)=\left(\begin{array}{l}
4 \\
1
\end{array}\right)+\left(\begin{array}{l}
3 \\
1
\end{array}\right)+\left(\begin{array}{l}
2 \\
1
\end{array}\right)+\left(\begin{array}{l}
1 \\
1
\end{array}\right)
\end{aligned}
$$

Where $x$ is a real number, define $[x] \equiv$ the greatest integer less than or equal to $x$. Property 3 is a symmetry property.

Property 3.

$$
\sum_{r=0}^{[n / 2]}\left[\begin{array}{c}
n \\
2 r
\end{array}\right]=\sum_{r=0}^{[n / 2]}\left[\begin{array}{c}
n \\
2 r+1
\end{array}\right]
$$

Example 3.

$$
\left[\begin{array}{l}
5 \\
0
\end{array}\right]+\left[\begin{array}{l}
5 \\
2
\end{array}\right]+\left[\begin{array}{l}
5 \\
4
\end{array}\right]=\left[\begin{array}{l}
5 \\
1
\end{array}\right]+\left[\begin{array}{l}
5 \\
3
\end{array}\right]+\left[\begin{array}{l}
5 \\
5
\end{array}\right]
$$


Analogous Property and Example:

$$
\begin{aligned}
\sum_{r=0}^{[n / 2]}\left(\begin{array}{c}
n \\
2 r
\end{array}\right) & =\sum_{r=0}^{[n / 2]}\left(\begin{array}{c}
n \\
2 r+1
\end{array}\right) . \\
\left(\begin{array}{l}
5 \\
0
\end{array}\right)+\left(\begin{array}{l}
5 \\
2
\end{array}\right)+\left(\begin{array}{l}
5 \\
4
\end{array}\right) & =\left(\begin{array}{l}
5 \\
1
\end{array}\right)+\left(\begin{array}{l}
5 \\
3
\end{array}\right)+\left(\begin{array}{l}
5 \\
5
\end{array}\right) .
\end{aligned}
$$

Property 4.

$$
\sum_{r=0}^{n}\left[\begin{array}{l}
n \\
r
\end{array}\right]=(n+1) \sum_{r=0}^{n-1}\left[\begin{array}{c}
n-1 \\
r
\end{array}\right]
$$

Example 4.

$$
\left[\begin{array}{l}
4 \\
0
\end{array}\right]+\left[\begin{array}{l}
4 \\
1
\end{array}\right]+\left[\begin{array}{l}
4 \\
2
\end{array}\right]+\left[\begin{array}{l}
4 \\
3
\end{array}\right]+\left[\begin{array}{l}
4 \\
4
\end{array}\right]=5\left\{\left[\begin{array}{l}
3 \\
0
\end{array}\right]+\left[\begin{array}{l}
3 \\
1
\end{array}\right]+\left[\begin{array}{l}
3 \\
2
\end{array}\right]+\left[\begin{array}{l}
3 \\
3
\end{array}\right]\right\}
$$

Analogous Property and Example:

$$
\begin{aligned}
& \sum_{r=0}^{n}\left(\begin{array}{l}
n \\
r
\end{array}\right) \\
& =2 \sum_{r=0}^{n-1}\left(\begin{array}{c}
n-1 \\
r
\end{array}\right) \\
& \left(\begin{array}{l}
4 \\
0
\end{array}\right)+\left(\begin{array}{l}
4 \\
1
\end{array}\right)+\left(\begin{array}{l}
4 \\
2
\end{array}\right)+\left(\begin{array}{l}
4 \\
3
\end{array}\right)+\left(\begin{array}{l}
4 \\
4
\end{array}\right)=2\left\{\left(\begin{array}{l}
3 \\
0
\end{array}\right)+\left(\begin{array}{l}
3 \\
1
\end{array}\right)+\left(\begin{array}{l}
3 \\
2
\end{array}\right)+\left(\begin{array}{l}
3 \\
3
\end{array}\right)\right\} .
\end{aligned}
$$

Property 5 follows from Property 4.

Property 5.

$$
\sum_{r=0}^{n}\left[\begin{array}{l}
n \\
r
\end{array}\right]=(n+1) !
$$

Example 5.

$$
\left[\begin{array}{l}
4 \\
0
\end{array}\right]+\left[\begin{array}{l}
4 \\
1
\end{array}\right]+\left[\begin{array}{l}
4 \\
2
\end{array}\right]+\left[\begin{array}{l}
4 \\
3
\end{array}\right]+\left[\begin{array}{l}
4 \\
4
\end{array}\right]=5 !
$$

Analogous Property and Example:

$$
\begin{gathered}
\sum_{r=0}^{n}\left(\begin{array}{l}
n \\
r
\end{array}\right)=2^{n} . \\
\left(\begin{array}{l}
4 \\
0
\end{array}\right)+\left(\begin{array}{l}
4 \\
1
\end{array}\right)+\left(\begin{array}{l}
4 \\
2
\end{array}\right)+\left(\begin{array}{l}
4 \\
3
\end{array}\right)+\left(\begin{array}{l}
4 \\
4
\end{array}\right)=2^{4} .
\end{gathered}
$$


For the nonnegative integers $x$ and $y$ where $x \leq y$, define $P_{x}^{y}$ to be

$$
P_{x}^{y} \equiv \frac{y !}{(y-x) !}
$$

Property 6.

$$
\sum_{m=0}^{n} P_{n-m}^{n+1} \sum_{r=0}^{m}\left[\begin{array}{c}
m \\
r
\end{array}\right]=\sum_{m=0}^{n} P_{n-m}^{n+1}(m+1) !=(n+1)(n+1) !
$$

Example 6. For $n=3$,

$$
\begin{gathered}
P_{3}^{4}\left[\begin{array}{l}
0 \\
0
\end{array}\right]+P_{2}^{4}\left\{\left[\begin{array}{l}
1 \\
0
\end{array}\right]+\left[\begin{array}{l}
1 \\
1
\end{array}\right]\right\}+P_{1}^{4}\left\{\left[\begin{array}{l}
2 \\
0
\end{array}\right]+\left[\begin{array}{l}
2 \\
1
\end{array}\right]+\left[\begin{array}{l}
2 \\
2
\end{array}\right]\right\}+P_{0}^{4}\left\{\left[\begin{array}{l}
3 \\
0
\end{array}\right]+\left[\begin{array}{l}
3 \\
1
\end{array}\right]+\left[\begin{array}{l}
3 \\
2
\end{array}\right]+\left[\begin{array}{l}
3 \\
3
\end{array}\right]\right\} \\
=4 \cdot 3 \cdot 2(1 !)+4 \cdot 3(2 !)+4(3 !)+(4 !)=4(4 !) .
\end{gathered}
$$

Analogous Property and Example:

$$
\sum_{m=0}^{n} \sum_{r=0}^{m}\left(\begin{array}{c}
m \\
r
\end{array}\right)=\sum_{m=0}^{n} 2^{m}=2^{n+1}-1
$$

For $\mathrm{n}=3$,

$$
\left(\begin{array}{l}
0 \\
0
\end{array}\right)+\left\{\left(\begin{array}{l}
1 \\
0
\end{array}\right)+\left(\begin{array}{l}
1 \\
1
\end{array}\right)\right\}+\left\{\left(\begin{array}{l}
2 \\
0
\end{array}\right)+\left(\begin{array}{l}
2 \\
1
\end{array}\right)+\left(\begin{array}{l}
2 \\
2
\end{array}\right)\right\}+\left\{\left(\begin{array}{l}
3 \\
0
\end{array}\right)+\left(\begin{array}{l}
3 \\
1
\end{array}\right)+\left(\begin{array}{l}
3 \\
2
\end{array}\right)+\left(\begin{array}{l}
3 \\
3
\end{array}\right)\right\}=2^{4}-1
$$

\section{Property 7.}

$$
\sum_{i=0}^{r}\left[\begin{array}{c}
n \\
i
\end{array}\right]=(n+1) \sum_{i=0}^{r-1}\left[\begin{array}{c}
n-1 \\
i
\end{array}\right]+n\left[\begin{array}{c}
n-1 \\
r
\end{array}\right]
$$

Example 7. For $n=6$ and $r=4$,

$$
\left[\begin{array}{l}
6 \\
0
\end{array}\right]+\left[\begin{array}{l}
6 \\
1
\end{array}\right]+\left[\begin{array}{l}
6 \\
2
\end{array}\right]+\left[\begin{array}{l}
6 \\
3
\end{array}\right]+\left[\begin{array}{l}
6 \\
4
\end{array}\right]=(6+1)\left\{\left[\begin{array}{l}
5 \\
0
\end{array}\right]+\left[\begin{array}{l}
5 \\
1
\end{array}\right]+\left[\begin{array}{l}
5 \\
2
\end{array}\right]+\left[\begin{array}{l}
5 \\
3
\end{array}\right]\right\}+6\left[\begin{array}{l}
5 \\
4
\end{array}\right]
$$

Analogous Property and Example:

$$
\sum_{i=0}^{r}\left(\begin{array}{l}
n \\
i
\end{array}\right)=2 \sum_{i=0}^{r-1}\left(\begin{array}{c}
n-1 \\
i
\end{array}\right)+\left(\begin{array}{c}
n-1 \\
r
\end{array}\right)
$$

For $n=6$ and $r=4$,

$$
\left(\begin{array}{l}
6 \\
0
\end{array}\right)+\left(\begin{array}{l}
6 \\
1
\end{array}\right)+\left(\begin{array}{l}
6 \\
2
\end{array}\right)+\left(\begin{array}{l}
6 \\
3
\end{array}\right)+\left(\begin{array}{l}
6 \\
4
\end{array}\right)=2\left\{\left(\begin{array}{l}
5 \\
0
\end{array}\right)+\left(\begin{array}{l}
5 \\
1
\end{array}\right)+\left(\begin{array}{l}
5 \\
2
\end{array}\right)+\left(\begin{array}{l}
5 \\
3
\end{array}\right)\right\}+\left(\begin{array}{l}
5 \\
4
\end{array}\right)
$$


Acknowledgment. The support from the U. S. Bureau of the Census to the author while serving as a research fellow is gratefully acknowledged.

\section{REFERENCES}

1. A. W. F. Edwards, Pascal's Arithmetical Triangle, Charles Griffin Books Series, Oxford University Press, New York , (1987).

2. R. L. Graham, D. E. Knuth, and O. Patashnik, Concrete Mathematics, Addison-Wesley, Reading, (1989).

3. D. E. Knuth, The Art of Computer Programming, Vol I, Seminumerical Algorithms, $2^{\text {nd }}$, Addison-Wesley, Reading, (1981).

4. B. Pascal, Traite du Triangle Arithmetique, Desprez(1665).

5. S. M. Ross, Introduction to Probability Models, $4^{\text {th }}$ Ed., Academic Press, San Diego, (1989).

6. H. S. Wilf, Combinatorial Algorithms: An Update, \# 55 in CBMS-NSF Regional Conference Series in Applied Mathematics, Society for Industrial and Applied Mathematics, Philadelphia, (1989).

7. T. Wright, A note on Pascal's triangle and simple random sampling, College Mathematics Journal, 20(1989)59-66.

8. T. Wright, Exact Confidence Bounds When Sampling From Small Finite Universes, Springer-Verlag, New York, (1991).

9. T. Wright, "Pascal's triangle gets its genes from Stirling numbers of the first kind", The College Mathematics Journal,(in press). 
ORNL/TM-12988

\section{INTERNAL DISTRIBUTION}

1. C. K. Bayne

2. J. J. Beauchamp

3. K. O. Bowman

4. C. C. Brandt

5. T. S. Darland

6. E. F. D'Azevedo

7. D. J. Downing

8. D. M. Flanagan

9. E. L. Frome

10. P. $\mathrm{Hu}$

11-15. M. R. Leuze

16. M. D. Morris

17. C. E. Oliver
18. G. Ostrouchov

19-23. S. A. Raby

24. C. H. Romine

25. R. L. Schmoyer

26-30. R. F. Sincovec

31-35. T. Wright

36. D. A. Wolf

37. Central Research Library

38. K-25 Applied Tech. Library

39. ORNL Patent Office

40. Y-12 Technical Library

41-45. Laboratory Records Department

46. Laboratory Records Dept. - RC

\section{EXTERNAL DISTRIBUTION}

47. Mx. Maxine Anderson-Brown, Program Manager, DIR, Room 2270-3, U.S. Bureau of the Census, Washington, DC 20233.

48. Dr. Dan Hitchcock, Office of Scientific Computing, ER-7, Applied Mathematical Sciences, Office of Energy Research, U.S. Dept. of Energy, Washington, DC 20585.

49. Dr. Fred Howes, Office of Scientific Computing, ER-7, Applied Mathematical Sciences, Office of Energy Research, U.S. Dept. of Energy, Washington, DC 20585.

50. Dr. David Nelson, Scientific Computing Staff, Applied Mathematical Sciences, Office of Energy Research, U.S. Department of Energy, Washington, DC 20585.

51. Office of Assistant Manager for Energy Research and Development, U.S. Department of Energy, Oak Ridge Operations Office, P.O. Box 2001, Oak Ridge, TN 37831-8600.

52. Office of Scientific and Technical Information, P.O. Box 62, Oak Ridge, TN 37830.

53. Dr. How J. Tsao, Management Services Division, Eastman Kodak, Rochester, NY 14652-3302.

54. Professor Mary F. Wheeler, Rice University, Department of Mathematical Sciences, P.O. Box 1892, Houston, TX 77251. 